\title{
The Advantages and Limitations of Low-Cost Single Frequency GPS/MEMS-Based INS Integration
}

\author{
Mustafa M. Amami * \\ Department of Civil Engineering, Benghazi University, Benghazi, Libya.
}

Global Journal of Engineering and Technology Advances, 2022, 10(02), 018-031

Publication history: Received on 27 December 2021; revised on 03 February 2022; accepted on 05 February 2022

Article DOI: https://doi.org/10.30574/gjeta.2022.10.2.0031

\begin{abstract}
Global Positioning System (GPS) provides a method for directly obtaining instantaneous position and velocity estimates using satellites-based passive range measurements. GPS is a whole day, all-weather, passive, satellite positioning system. Inertial Navigation System (INS) is a navigation aid system that uses a computer and Inertial Measurement Unite (IMU). IMU includes motion sensors and rotation sensors to continuously calculate relative position, orientation, and velocity. The integration of GPS/INS can help to overcome the limitations of the two systems providing integrated system better than either on a stand-alone basis. The integration of low-cost INS with dual frequency GPS has been widely studied and the same for the integration of tactical grid INS with low-cost GPS. However, during the last a few years, a number of low-cost GPS \low-cost INS integrated systems have been introduced and become popular in various engineering applications. However, reliable investigations into the advantages, limitations and quality of such integration level are still needed and more efforts are required, which will be the focus of this paper.

The methodology followed in this paper for evaluating the integration of low-cost GPS/INS sensors depends on evaluating the two sensors individually and comparing the results with the integrated system. The results show that low-cost single frequency GPS receivers are able to provide a comparable accuracy level in both static and kinematic carrier phase differential GPS (DGPS). As for low-cost Micro-Electro-Mechanical System (MEMS)-based IMU, the accelerometers have provided instability comparing to gyros. The performance of gyros can be improved based on modelling the nearly-linear behaviour of the gyro drift. Tests show that the integration of low-cost GPS sensors with MEMS-based INS degrades the quality of gyro measurements and may not add any improvements to the quality of the individual GPS positioning.
\end{abstract}

Keywords: Low-cost GPS/INS integration; MEMS-IMU; Code positioning; Single frequency DGPS

\section{Introduction}

Global Positioning System (GPS) provides a method for directly obtaining instantaneous position and velocity estimates using satellites based passive range measurements. GPS is a whole day, all-weather, passive, satellite positioning system. It provides high accuracy, instantaneous position, and time information across the world. GPS satellites transmit two Lband frequencies, namely: L1 (1575.42MHz) and L2 (1227.6MHz) with carrier wavelengths of approximately $19 \mathrm{~cm}$ and $24 \mathrm{~cm}$, respectively. Two codes are modulated onto the carriers, namely: the Coarse/Acquisition (C/A) code and the Precise (P) code. The first is modulated onto the L1 carrier only with a wavelength of nearly $300 \mathrm{~m}$, whereas the second has a wavelength of $30 \mathrm{~m}$ and modulated onto both L-band frequencies. In addition to these two codes, the system transmits a navigation message including the satellite ephemeris, satellite clock coefficients, satellite health data and ionosphere modeling factors. Almanac is also transmitted to help the receiver in finding out the satellites reducing the

\footnotetext{
* Corresponding author: Mustafa Amami

Department of Civil Engineering, Benghazi University, Benghazi, Libya.

Copyright $(2022$ Author(s) retain the copyright of this article. This article is published under the terms of the Creative Commons Attribution Liscense 4.0.
} 
searching time. GPS has a number of ground-based control stations for monitoring the satellites, determining the satellite orbits, and uploading the navigation messages [1, 2]

The GPS pseudo-range between receiver and satellite is obtained by matching the satellite code with the internal code generated by the receiver and scaling the time difference by the speed of light. Pseudo-range GPS C/A code observables can provide absolute stand-alone positioning with 3D accuracy of a few meters. Stand-alone GPS C/A code positioning needs at least four satellites to solve the four unknowns of each epoch (the 3D coordinates of the position and the receiver clock time) [4]. The limited accuracy of this positioning technique is attributable to different error sources, including: satellite errors (clock and orbit), propagation errors (ionosphere, troposphere, and multipath), and receiver errors (clock, measurements noise, and phase center variation). Stand-alone GPS C/A code positioning can be used for a wide range of engineering applications not requiring high accuracy, such as car navigation, approximate positioning, Unmanned Aerial Vehicle (UAV), mapping for GIS applications, agriculture, disaster relief, emergency, low-cost Mobile Mapping Systems (MMS), and tours [3, 4, 5].

The carrier phase observation is formed by stripping the code from the received signal. Carrier phase observation can be measured to the level of 0.01 cycles giving millimeters accuracy. Just the fractional phase with the accumulated integer number of wavelengths can be measured by the receiver as the connection between the satellite and receiver is available. As for the initial total number of integer wavelengths, it is unknown which makes the absolute standalone one epoch based positioning impossible for carrier observations. This initial unknown number is known as the integer ambiguity. Differencing GPS (DGPS) observations can be used for solving this problem providing precise relative positioning. Relative positioning aims at determining the coordinates of an unknown point with respect to a known point or determining the vector between the two points and this requires simultaneous observations at the two points. With DGPS, some of GPS errors are reduced or removed based on the high correlation between these errors over short baselines. Differencing observations can be formed using code or carrier phase taking one of the following forms: single, double and triple differences. Single differences can be formed between two receivers, two satellites, or two epochs. Double differences are formed between any two single differences, whereas triple differences are between the three forms of single differences, including two receivers, two satellites, and two epochs $[1,2,6]$.

Carrier phase DGPS can be formed using single frequency (L1) or dual frequency (L1\&L2). The dual frequency GPS receivers are high-cost comparing to the single frequency receivers as they can deal with ionosphere error providing accuracy of millimeters level. Such rate of positioning quality can be used in precise applications, such as cadastral surveying, geodetic control, and strictures deformation. Low-cost GPS receivers (single frequency) can also provide carrier phase DGPS positioning based on L1 frequency; but the accuracy level tends to be degraded with increasing the baseline length as the ionosphere error becomes significant. Single frequency GPS receivers have become more desirable due to their low cost and used in a wide range of engineering applications, such as mapping, geo-referencing, GIS data collection, aerial and close range photogrammetry, and transportation [1, 6] . Figure (1) shows examples of low-cost single frequency GPS receivers.

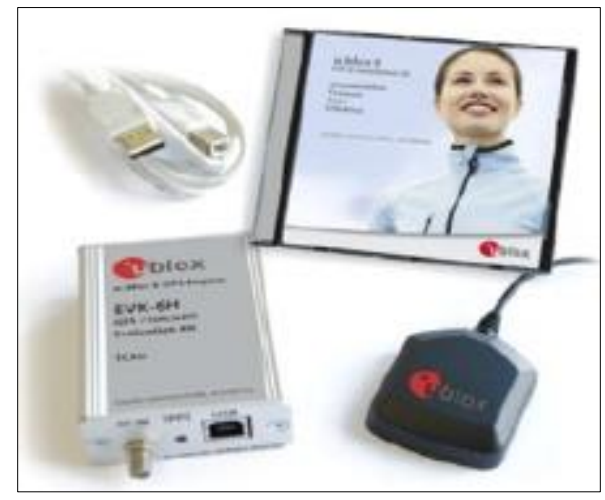

Figure 1 u-blox GPS receiver

Inertial Navigation System (INS) is a navigation aid system that uses a computer, motion sensors and rotation sensors to continuously calculate the position, orientation, and velocity relative to a known starting point. The basic idea behind INS is to integrate acceleration and rotation measurements into relative speed of movement and direction of a moving object without the need for external references. Modern IMUs consist of three orthogonally mounted gyroscopes and accelerometers, measuring angular velocity and linear acceleration, respectively. Three magnetometers tend to be added to this system for bounding the significant drift of low-cost gyroscope with time [2, 7]. 
The accuracy of INS depends mainly on: the initial state accuracy, inertial sensor quality, such as accelerometers and gyros, and calculation accuracy including corrections. Also, the accuracy tends to be a function of the cost which increases hand by hand with increasing the stability and reliability of inertial sensors. INS is a self-contained navigation passive, worldwide, easy to operate and independent system and it can be used in all weather and attitude. However, INS should be provided with initial position and rotations for achieving absolute orientation. When it has been initialized, no more help is needed for navigation. INS has become a necessary request in a great deal of application, such as the aircraft navigation, submarines and ships, tactical and strategic missiles and spacecraft. Current developments in the Micro Electro Mechanical System (MEMS) construction of devices lead to manufacturing undersized and light IMUs opening the doors for such system to be used in more applications, such as human and animal motion capture [3]. Examples of low-cost MEMS-based INS are shown in figure (2).

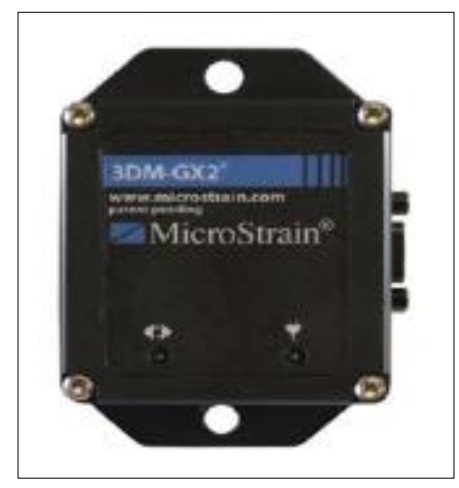

Figure 2 Microstrain IMU

INS suffers from different type of errors, some of them can be bounded, such as those of acceleration, velocity and initial tilt, and others hard to be bounded including azimuth misalign, leveling gyro drift and azimuth gyro drift. Small errors in the acceleration and angular velocity measurement are cumulated with time to be great errors in position where each position is calculated from the previous calculated position. Therefore, the position must be regularly updated from another navigation system depending on the quality of the sensors used and the accuracy required from the system [2, 7].

INS, based on MEMS technology, has become commonly used due to the significant low-cost, tiny size and not including any spinning wheels. As a result, noise, inertial forces and mechanical failures can be avoided. MEMS based gyros have many advantages over conventional gyros, such as power independent memory, very low power consumption, not including bearings, lubricants or fluid, very short start up time, and very rugged and reliable. On the other hand, they are very sensitive to temperature changes, analogue output requires sampling, high gyro drift rates (20 to 30 degrees/hour), and not accurate enough for higher performance applications [2].

The integration between GPS and INS can help to overcome the limitations of the two systems providing integrated system better than either on a stand-alone basis. For example, INS position error drifts with time, whereas GPS solution is time independent. Also, INS outputs are relatively high frequency, whereas GPS solution is low frequency. INS is totally self-contained and autonomous operation, while GPS is dependent on the availability of satellites. Attitude capability is limited in the case of GPS comparing to INS which can provide accurate and high rate attitude data. The need of initialization is another limitation of INS where it just provides relative positioning and rotations. This is not the case with GPS which can self-initialize in flight. In the integrated system, INS aids GPS to reduce susceptibility to jamming, sensitivity to vehicle manoeuvres, velocity errors and satellite acquisition and reacquisition times. On the other hand, GPS helps INS to reduce propagation of errors with time and to provide initial positioning and rotating. This integration can be carried out in one of three main integration levels, namely: uncoupled, loosely coupled, and tightly coupled [2, 3, 7].

Uncoupled integration is the simplest level of integration as the INS indicated position and velocity are reset at regular intervals of time using the position and velocity estimated by GPS. This method engages minimum changes to both systems and it does not help to enhance the performance and avoid jamming. Also, when GPS is hidden, the quality of positioning solution decreases rapidly $[2,7]$. The loosely coupled integration is the typical integration of stand-alone INS and GPS. In this integration level, the GPS is run autonomously and, at the same time, INS and GPS integrated solution is enabled. The estimated position and velocity, provided by INS and GPS are compared and the differences are inputted to the estimation filter. The advantage of this approach comes from its redundancy where two navigation solutions are 
provided: that of stand-alone GPS and the other of GPS/INS integration. This integration approach can be used with any INS and GPS receiver if the necessary number of GPS satellites is available. Also, loosely integration has high flexibility and modularity as well as less computation and complexity due to the independent operation. When GPS is hidden or less than the necessary number of satellites are available, the INS stand-alone solution based on Kalman filter is used to fill in the gap which will drift in time depending on the stability of the accelerometers and gyros used [2]. The tightly coupled integration is another GPS/INS integration method, in which no separated GPS navigation solution is given. In this method, a single integration filter is used to combine the raw GPS measurements and those constructed from INS prediction. The filter straight accepts their differences to get the INS error estimates. This integration gives a more accurate solution than the previous methods. This can be attributed to the fact that the GPS observables used in the combination process of the tightly coupled integration are not in the same correlation level of the position and velocity solutions used in the loosely coupled approach [7].

In this paper, the integration of low-cost single frequency GPS with low-cost MEMS based INS will be evaluated in order to investigate whether this integration can help to overcome the limitations of the two systems and provide integrated system better than either on a stand-alone basis. This level of integration has become more and more common and a number of integrated systems have been introduced during the last a few years. Figure (3) show an example of low-cost GPS/INS integrated sensors.



Figure 3 Xsens low-cost GPS/INS system

The integration of low-cost INS with dual frequency GPS has been widely studied and the same for the integration of tactical grid INS with low-cost GPS. However, reliable investigations into the integration of the two low-cost level sensors are still needed and more efforts in this area are required, which will be the focus of this paper.

\section{Methodology}

The methodology followed in this paper for evaluating the integration of low-cost GPS/INS sensors depends on evaluating the two sensors individually, and comparing the results with those of the integrated system Firstly, the lowcost single frequency GPS receiver will be tested individually in different GPS environments with different positioning techniques to investigate the accuracy level can be obtained from such sensor.

Secondly, low-cost MEMS based INS will also be tested individually to investigate the gyros drifting rates and the accelerometers performance. After that, the integration of these two low-cost sensors will be evaluated comparing to the stand-alone performance of each sensor.

\section{Evaluating Low-Cost Single Frequency GPS Receivers}

u-blox GPS receivers are considered to be from the most common low-cost L1 frequency (C/A Code) GPS receivers which are used in a great range of navigation and positioning applications. These receivers can provide an estimated accuracy of 2.5 and $5 \mathrm{~m}$ in plan and height, respectively. According to the manufacturer, this small and light receiver, with size of $25.4 \mathrm{~mm} \times 25.4 \mathrm{~mm} \times 3 \mathrm{~mm}$ and weight of just 3 grams, has several advantages, such as excellent navigation accuracy even at low signal level, powerful multipath detection and removal, fast time to first fix, high acquisition and tracking sensitivity, ultra-low power consumption and industrial operating temperature range between -40 and $85^{\circ} \mathrm{C}$. This is in addition to its ability to be supported by DGPS networks, such as WAAS and EGNOS providing comparable accuracy level. u-blox GPS receiver performs the whole GPS signal processing in one receiver with Patch antenna providing uncomplicated and effortless integration with short time, low-cost and minimum design risks [2]. 
Two common low-cost u-blox GPS receivers (u-blox 4 \& u-blox 6) have been chosen in this paper to be tested and evaluated in different GPS environments with different positioning techniques. The two receivers have been connected with dual frequency GPS receiver (Leica GS10) to the same vertical dipole antenna by antenna splitter. Using the same antenna and applying the tests at the same time help the receivers to be evaluated under the same GPS conditions, such as satellite geometry and multipath environment. Leica Geo Office 8.3 software has been used to process the raw data of each receiver as static carrier phase DGPS. This software needs the u-blox files to be converted to RINEX format using, for example, Teqc software. The coordinates achieved from each receiver have been compared to the 'true' coordinates achieved via dual frequency GPS receiver with several hours of static carrier phase DGPS. Different periods of static carrier phase DGPS have been tested to investigate the effect of fixing time on the positioning quality. Figure (4) shows the 3D positioning accuracy as a function of fixing time.

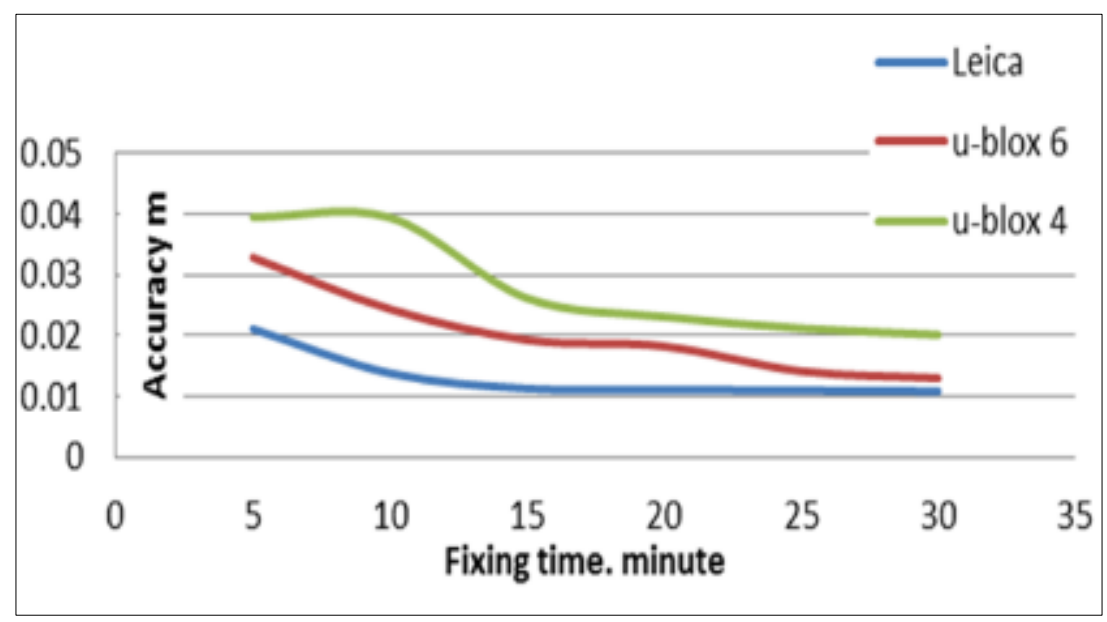

Figure 4 Static carrier phase DGPS positioning accuracy as a function of fixing time

It is clear from the figure that the accuracy, in general, is a function of fixing time where the more observations, the better outputs. This can be attributable to the fact that increasing the number of observations for measuring the same variables tends to close the final results to the absolute mean value which is achieved using infinity number of observations. This absolute mean value is equal to the true value when cancelling out the systematic errors. From the figure, the positioning quality provided by the two single frequency GPS receivers is less than that of dual frequency receiver. This can be referred to the following reasons:

- Dual frequency receivers have the ability to mitigate the effect of ionosphere delay through a linear combination of code or carrier measurements.

- Dual frequency receivers has the ability to mitigate the effect of multipath using powerful and complex technique called narrow correlation, whereas the low-cost GPS receivers used in this test are provided with simple mitigation filters and depend mainly on the recommended Patch antenna to eliminate the effect of the signals reflected from low angles.

- Using dual frequency receiver as a base station allows for better differential processing where the measurements of the two receivers are more precise due to the ionosphere and multipath mitigation techniques.

Also, it can be seen from the figure that the accuracy of the Leica receiver has taken the shortest fixing period to be nearly constant. This is a clear indication about the precision degree of the dual frequency observations comparing to L1 based receivers. With precise measurements and neglected systematic errors, small number of observations can provide results close to the absolute mean value and increasing the number of such precise observations makes the outputs more reliable and the change in the accuracy level tends to unnoticeable.

u-blox 6 has given the second best results close to those of Leica. This is because with carrier phase DGPS, even using single or dual frequency receivers, some GPS errors are eliminated, such as satellite orbit and clocks, and others are mitigated, such as ionosphere and troposphere delays. These last depend on the baseline and the difference in height between the base station and the rover which have been within $20 \mathrm{~m}$ and a few $\mathrm{mm}$, respectively in this test. However, the dual frequency receiver is still better due to the reasons mentioned above. 
The differences between receivers have been in centimeters level with short fixing time and getting smaller with longer period. This might be because the observations of single frequency receiver are, theoretically, less precise than those of dual frequency due to the ability of the last to mitigate the effect of ionosphere delay significantly and reduce the effect of multipath using narrow correlation technique. As a result, with small number of observations, the differences between the two receivers can be clear. However, with increasing the number of observations, the accuracy of the less precise measurements increases significantly compared to that of precise measurements which might improve slightly. The other theoretical reason behind the less accurate results of the low-cost receivers is the receiver noise which is often significant in such receivers [1]. This GPS source error cannot be reduced or cancelled out with DGPS rounding about one centimeter. However, the receiver noise of the low-cost GPS receivers used in this project has been evaluated, as will be shown later, and found as small as can be neglected.

It is also clear from the figure that u-blox 6 has been better than u-blox 4 to some extent. According to the manufacturer, u-blox 6 receiver has been developed for better positioning but these developments have not been mentioned in the manual. The only clear reason behind these differences in the results might be the number of channels of each receiver, where u-blox 6 is provided with 50 channels comparing to 16 channels for the other receiver. In addition to reducing power consumption, increasing the number of channels helps to speed up satellite acquisition, increase the sensitive for GPS signals and reduce the probability of losing a 3D fix even in urban and dense areas. All of these advantages can help to provide better positioning accuracy [2].

For more investigations, the same data have been processed as kinematic carrier phase DGPS to evaluate the accuracy of each receiver with mobile solution. The position of each epoch has been compared to the antenna position and the Roth Mean Squares Error (RMSE) has been calculated. Moreover, code measurements of the two low-cost receivers have been evaluated to assess the quality of stand-alone code positioning which is one of the main navigation options adopted in low-cost integration systems. Table (1) illustrates the results.

Table 1 u-blox 6 Vs. u-blox 4: KDGPS \& A/C Code

\begin{tabular}{|c|c|c|c|c|c|c|}
\hline Positioning technique & \multicolumn{3}{|c|}{ u-blox 6 (RMSE) } & \multicolumn{3}{c|}{ u-blox 4 (RMSE) } \\
\cline { 2 - 7 } & E m & N m & H m & E m & N m & H m \\
\hline KDGPS & 0.043 & 0.049 & 0.084 & 0.047 & 0.058 & 0.109 \\
\hline A/C Code & 1.087 & 1.163 & 1.972 & 1.191 & 1.312 & 2.400 \\
\hline
\end{tabular}

It can be seen from the table that $\mathrm{u}$-blox 6 receiver is better than $\mathrm{u}$-blox 4 in both kinematic carrier phase DGPS and A/C code positioning, which can be attributed to the above mentioned reasons. It is clear from the table that, in general, the plan quality is better than the altitude quality which can be referred to the satellite geometry. Theoretically, the best overall quality can be achieved with 4 satellites distributed with 90 degrees in azimuth and at 40 to 50 elevation angle. Increasing this last helps to achieve better plan quality and leads the vertical quality to be reduced and vice versa [2].

On the other hand, using low elevation satellites tends to be avoided affecting the attitude quality. This is because GPS signal path of the low elevation satellite passes through more atmosphere than the vertical satellite. This is important where the positioning calculation in GPS is based on the assumption that GPS signal travels in a vacuum. Therefore, signals of low elevation satellites have more delay and consequently give less precise results. Also, passing the signal through the atmosphere for longer distances tends to make it noisier and not clean affecting the goodness of data. Satellite geometry can also be the reason behind being the quality in E direction, generally, better than $\mathrm{N}$ direction as seen from the table. In GPS, the number of satellites in E-W direction is more than that of N-S direction due to the inclination angle of the satellite orbits. GPS satellite orbits have 55 inclination angles which mean the satellites fluctuate in the area between +55 degree and -55 degree from the Equator [1].

This means that in areas located above this degree, the majority of satellites are locate overhead and on E, W and S directions and a few satellites can be detected in the north with low elevation angles due to the height of the satellite above the Earth. Figure (5) shows the ground track of the satellite with an inclination angle of $45^{\circ}$, figure (6) shows an example of the satellite distribution over Nottingham University and figure (7) illustrates how the satellites appear in the North. 




Figure 5 Satellite ground track

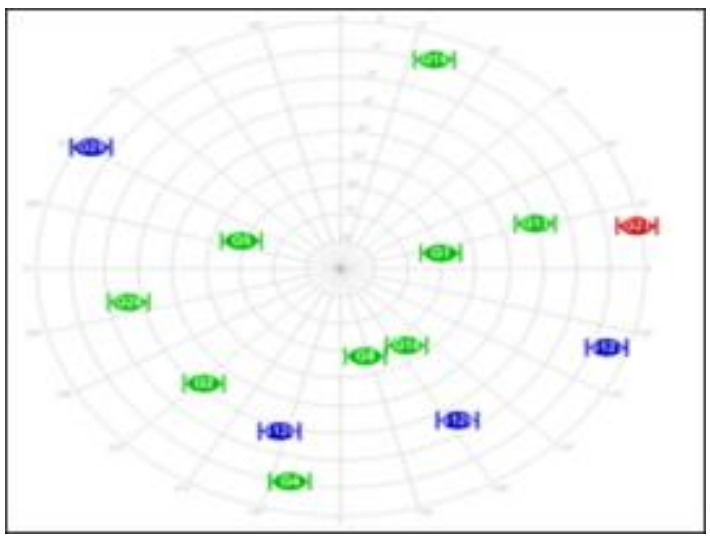

Figure 6 GPS satellite distribution



Figure 7 Satellites in North direction

Another test has been applied to investigate the possibility of using such low-cost GPS receivers as a base and rover for single frequency DGPS. This can help to reduce the cost significantly where the user just needs one known point within a few Kilometers from the working area and then low-cost static and kinematic carrier phase DGPS can be applied. Two u-blox 6 GPS receivers have been fixed on known points and the raw data has been collected for different periods and static and kinematic DGPS have been applied using GravNav software. Leica dual frequency receiver has also been used as a base station in this test to investigate the effect of using different types of receivers as a base station. Figure (8) and table (2) illustrate the results.

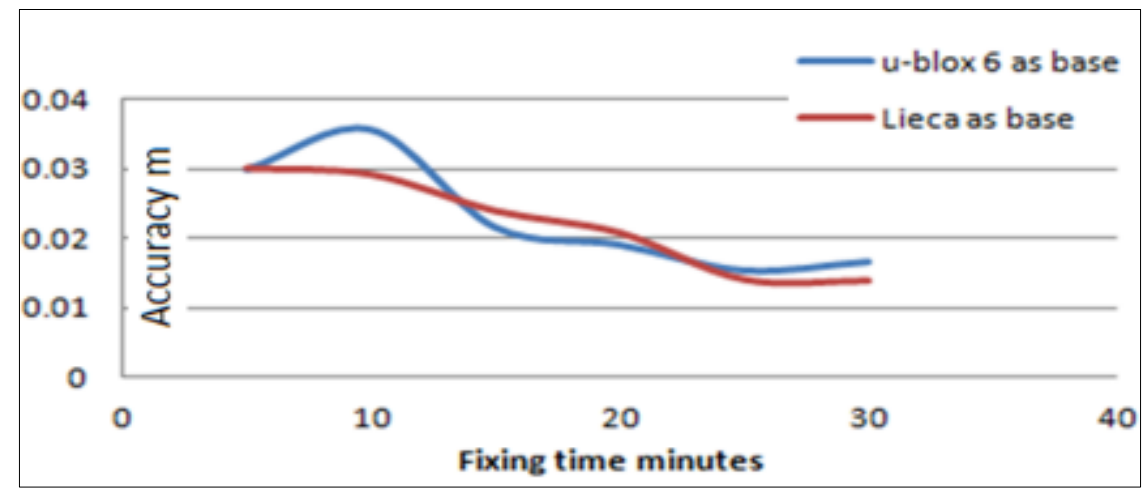

Figure 8 Static carrier phase DGPS with low-cost GPS receivers as a rover and a base station 
Table 2 Using Leica and u-blox 6 as a base station for kinematic carrier phase DGPS (RMSE)

\begin{tabular}{|c|c|c|c|c|c|}
\hline \multicolumn{2}{|c|}{ u-blox receiver } & \multicolumn{3}{c|}{ Leica receiver } \\
\hline $\mathrm{E} \mathrm{m}$ & $\mathrm{N} \mathrm{m}$ & $\mathrm{H} \mathrm{m}$ & $\mathrm{E} \mathrm{m}$ & $\mathrm{N} \mathrm{m}$ & $\mathrm{H} \mathrm{m}$ \\
\hline 0.037 & 0.048 & 0.091 & 0.042 & 0.045 & 0.078 \\
\hline
\end{tabular}

The results show that an accurate single frequency DGPS can be applied using low-cost single frequency GPS receivers as a rover and a base station, in both: static and kinematic positioning. It should be mentioned that when using single frequency receiver as a rover, just L1 measurements are used from the base station file for applying carrier phase DGPS even if dual frequency receiver is used. This logically means there should be no differences in the results between utilizing different receivers as a base station. However, the results illustrate some differences which might be attributed to the differences in the two raw data files where GPS observations tend to be filtered in the receiver before recorded and these filters are different between receivers based on the required quality and applications.

As mentioned above, the receiver noise is one of the GPS error sources not mitigated or cancelled out with the carrier phase DGPS and can be doubled. To investigate the level of these low-cost receiver noise, all errors, except receiver noise, should be cancelled out. To do that, zero baseline test has been applied using GPS simulator. This guarantees that all GPS source errors are cancelled out with static DGPS, except the receiver noise. Zero baseline test using GPS simulator overcomes the outdoor zero baseline tests in terms of cancelling out the antenna noise effect. Two u-blox 6 GPS receivers have been tested for 12 hour to be tested with all possible satellite constellations. Zero baseline test has been carried out between the two receivers, each one as a rover and a base station and the outputs have been comparing to the true position chosen in the simulator. The results show that the noise levels of the two receivers are within 1 to 2 millimeters as illustrates in table 3.

Table 3 u-blox 6 GPS receiver noise level (RMSE) mm

\begin{tabular}{|l|l|l|l|}
\hline Receiver & Test 1 & Test 2 & Test 3 \\
\hline No. 1 & 1.584 & 1.335 & 1.530 \\
\hline No. 2 & 1.437 & 1.646 & 1.438 \\
\hline
\end{tabular}

According to [4], in the case of the low cost receiver, the carrier phase observation is expected to be slightly degraded compared to that recorded using a higher grade receiver, perhaps with an increased noise level due to lower grade components. Increasing system noise on the dynamic range of the carrier tracking loop can affect a carrier phase pseudo-range observable giving a greater number of cycle slips due to signal loss and could result in greater noise levels due to the need to increase tracking loop bandwidth.

In conclusion, in this section, low-cost single frequency GPS receivers have been tested to assess their performance as an initial step to be tested with the integration system. The receivers have been tested in different GPS environments with different positioning techniques. Tests show that low-cost GPS receivers, such as those tested, are able to provide a comparable positioning accuracy level in both static and kinematic DGPS solutions. The results show the ability of such sensors to provide accuracy of $1 \mathrm{~cm}$ with static carrier phase DGPS and a mobile solution with 5 and $10 \mathrm{~cm}$ accuracy in plan and height, respectively. Furthermore, the tests show also the high possibility of these receivers to be used as a rover and a base station to carry out low-cost static and kinematic DGPS. In terms of A/C code positioning, 3D accuracy of a few meters can be obtained, deceasing significantly in multipath GPS environments.

\section{Evaluating Low-Cost Inertial Navigation System (INS)}

The Microstrain 3DM-GX3-25 Inertial Measurement Unit (IMU) used in this paper is inexpensive vibrating structure gyroscopes manufactured with MEMS technology. This sensor, according to the manufacture, can provide attitude heading range of 360 degree about all 3 axes with static accuracy of 0.5 degree and dynamic accuracy of 2 degrees. Also, the sensor with its amazing size and weight can be used in operating temperature between $-40 \mathrm{o}$ and $70 \mathrm{o}$ with a gyro 
drift rate of 0.25 degree/second. In this section, the sensor will be tested to evaluate the rotations quality and to investigate the possibility of improving the outputs by studying, modeling and correcting the gyro angular drifts.

The sensor has been connected to a data logger and tested several times as stationary for about an hour. Figure (9) illustrates an example of the drifts of Pitch angle, from different tests as a function of time. The results show that the Euler angles drift with time, in average, by $0.29 \mathrm{deg} . / \mathrm{sec}$, which is close to that mentioned by the manufacturer. This drift rate is good compared with other low-cost IMUs, such as Inertial-Cube3 and Honeywell 3000, which have been tested beside this sensor giving drift rate of nearly $1 \mathrm{deg}$./sec when stopping the help of magnetometers. It can be seen also that the general trend of drifts, in all tests, increases quasi-linear but with different slops which is useful for modeling and correcting the drifts. Gyros and accelerometers data collected during these tests have also been processed to calculate the relative positioning accuracy level can be provided by the sensor. The results have reflected instability in the performance of accelerometers and considerable random changes in the position although the sensor is stationary.



Figure 9 Low-cost gyro drifts (Pitch angle)

Based on the linearity of the general tendency of drifting, a simple linear filter has been designed to provide more precise rotations. The idea of the filter is based on determining the average drift rate of each angle using the best fit line and least squares and using this rate as a correction for the following rotations. When the sensor is stationary, the change in Euler angles should be zero. The average drift rate for each angle has been calculated based on the observations of the first 10s and used to correct the rest of data. Results show amazing improvements in the gyro drifts where the errors are reduced to be less than $0.05 \mathrm{deg}$./sec. Figure (10) illustrates the drift rate before and after applying the linear filter and Figure (11) shows the results of several tests carried out to investigate the ability of this filter for bounding the gyro drifts.

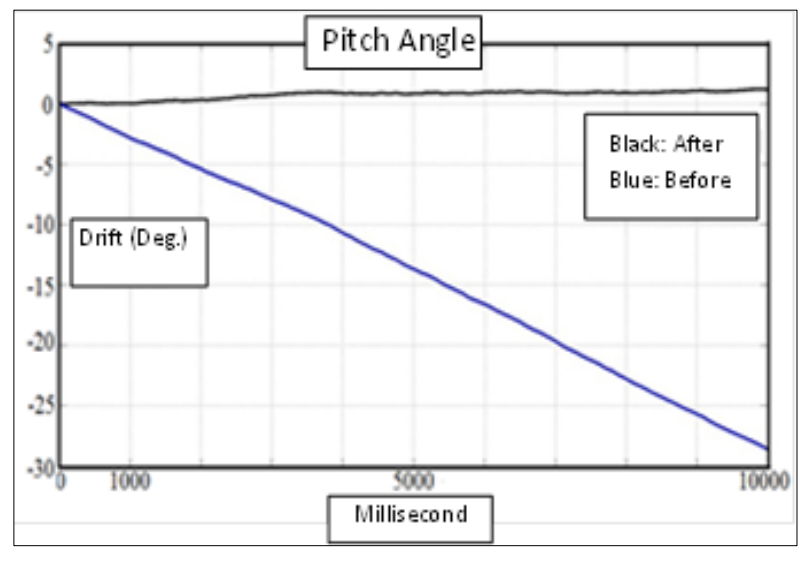

Figure 10 Gyro drifts before and after applying the linear correction on Pitch angle

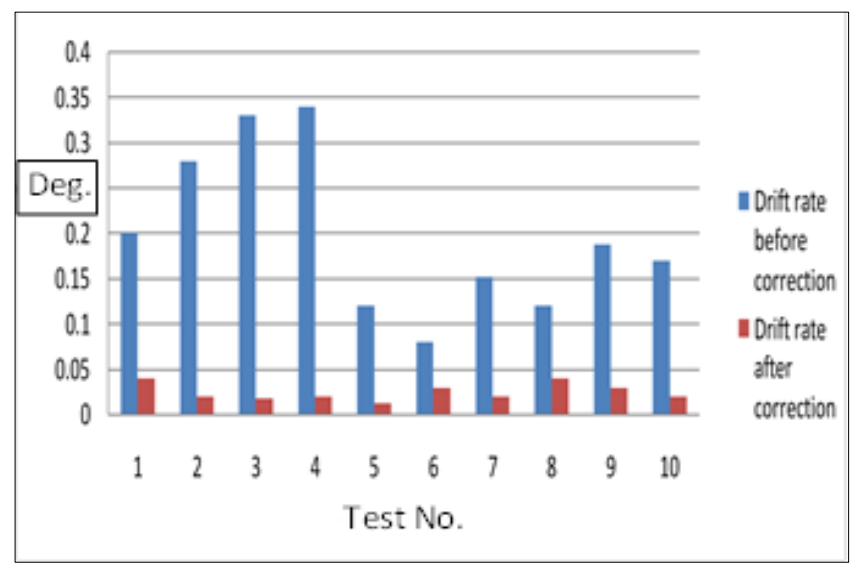

Figure 11 Drift rate in Pitch before and after filtering 
In conclusion, Microstrain 3DM-GX3-25 IMU used in this project has been evaluated in terms of rotation quality, drift rate, and modeling and correcting gyro drifts. Tests show that the sensor can provide drift rate of about $0.29 \mathrm{deg} / \mathrm{sec}$ and the angular drift of this sensor is quasi-linear. Instability in the performance of accelerometers has been recorded during the tests providing considerable errors even in stationary case. Simple linear filter has been designed and used to correct the angular drifts reducing the drift rate to round about $0.05 \mathrm{deg} . / \mathrm{sec}$.

\section{Evaluating the Integration of Low-Cost GPS/INS Sensors}

In this section, the integration between the two low-cost GPS and INS sensors, tested above, is evaluated. NovAtel GPS/INS system, u-blox 6 GPS receiver, Microstrain 3DM-GX3-25 IMU, and data logger have been fixed in the GPS/INS testing van of Nottingham University. NovAtel system is used as a reference where it includes dual frequency GPS receiver and high precise tactical IMU. In addition to comparing the low-cost navigation solution to that of NovAtel, the individual performance of the low-cost GPS receiver as well as IMU will be compared with the low-cost navigation solution to evaluate whether such integration is useful for the two sensors. Kinematic data for about an hour has been collected in different GPS environments such as open sky, between buildings and under dense trees. Also, the GPS antenna has been switched off in some areas for testing the sensors when GPS signals are completely hidden.

The data of NovAtel system have been loaded to GrafNav software to be integrated tightly with the row data of the Nottingham Geospatial Institute base station. This is because tightly coupled (TC) does not need full GPS solution to achieve a full navigation solution and it can provide more precise results than the other integration levels with precise IMU. The same base station data has been used with the low-cost GPS and INS data which have been integrated loosely (LC) and tightly (TC) and compared to NovAtel results. Figure (12) shows examples of the results of low-cost GPS/INS integration compared to NovAtel system.

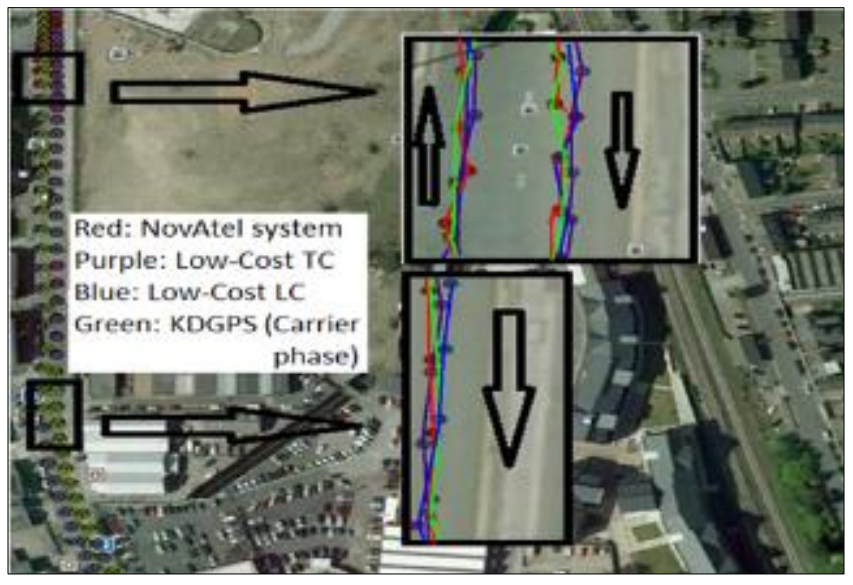

Figure 12 GPS/INS integration in Open sky

As seen from figure (12), in open sky where a significant number of satellites is available, the low-cost GPS/INS integration has provided excellent positioning results close to that of NovAtel using both TC and LC integration levels (RMSE of $3.281 \mathrm{~cm}$ and $3.301 \mathrm{~cm}$, respectively). This is because when GPS is available and healthy, the navigation solution in the case of low-cost loosely coupled GPS/INS integration depends completely on GPS carrier phase and partially on code measurements for determining the receiver position and uses the INS measurements to fast the signal reacquisition. In the case of tightly coupled integration, the measurements of GPS and INS are solved together based on the inputted weights to determine the receiver position. Therefore, with enough number of satellites and small INS weights, as in the case of the low-cost IMU used in the test, the effect of the IMU measurements on the tightly coupled navigation solution will be insignificant. Also, the quality of the achieved results can be referred to using $10 \mathrm{~Hz}$ GPS data rate. This helps to reduce the updating periods of the IMU and consequently better results can be adopted from the navigation solution which takes the IMU data rate.

When the number of satellites decreased due to the dense tress as shown in figure (13), the loosely coupled navigation solution has degraded comparing to that of tightly solution. This is because when GPS measurements are not adequate for getting 3D solution, loosely coupled solution depends on the IMU measurements smoothed by Kalman filter to fill in the gaps which are degraded rapidly due to the instability of accelerometers and gyros. However, the tightly coupled solution uses the available GPS measurements (even if they are not enough for providing a full GPS solution) with the IMU observations which keep the solution better. 


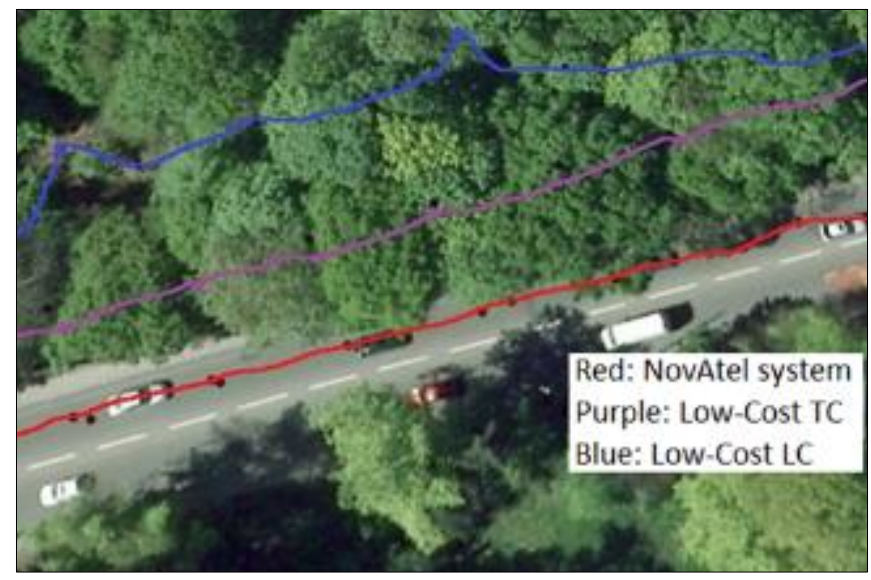

Figure 13 GPS/INS integration with limited satellites

When GPS is hidden under very dense tress or when switching the antenna off, the low-cost loosely and tightly solutions are degraded significantly comparing to NovAtel system as shown in figure (14). This is expectable because of the high and fast drifting rates of the low-cost gyros and accelerometers. These drifts grow even in stationary case reaching several degrees and meters per seconds for the gyros and accelerometers, respectively. In the dynamic case, the drifts tend to be considerable, especially in the case of accelerometers where any vibration in the platform is translated as change in the position.



Figure 14 GPS/INS integration when GPS is hidden)

Moreover, it can be noted that although the two solutions depend mainly on the INS measurements when GPS is hidden, the behavior of the two navigation solutions are completely different. This is because the prediction and smoothing steps in Kalman filter depend completely on the inputted and used measurements which are different in the two cases as GPS and IMU measurements are used together in TC solution. Also, it can be seen that the navigation solution, when GPS is hidden, depends on the trajectory direction, as seen in figures (15\&16). In the straight path, the solution has been acceptable for a good period before start drifting. However, in the case of curvy path, the solution starts drifting directly and significantly. This is because the prediction step in Kalman filter is affected considerably by the behavior of the latest updated measurements and tends to follow the same manners. The higher rotating and horizontal vibrating levels in the case of curvy paths can also affect the quality of the IMU measurements comparing to straight path.

In curvy paths, the horizontal vibration and suddenly changing in the velocity are translated as changing in the sensor's positions. The gyro drift can also play a role in these errors where the vibrations are considered as angular velocities. These last are integrated and translated as changing in the sensor's directions. The gyro drifts can be seen as limited in terms of value but they have a significant effect on the navigation solution where rotations are used hand by hand with accelerations to determine the relative positioning of the IMU. 


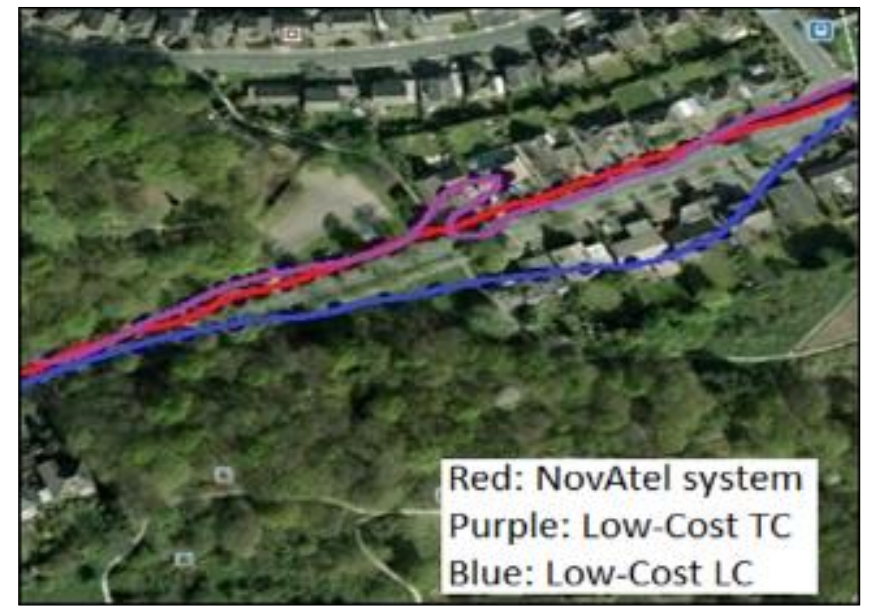

Figure 15 GPS off (Straight path)

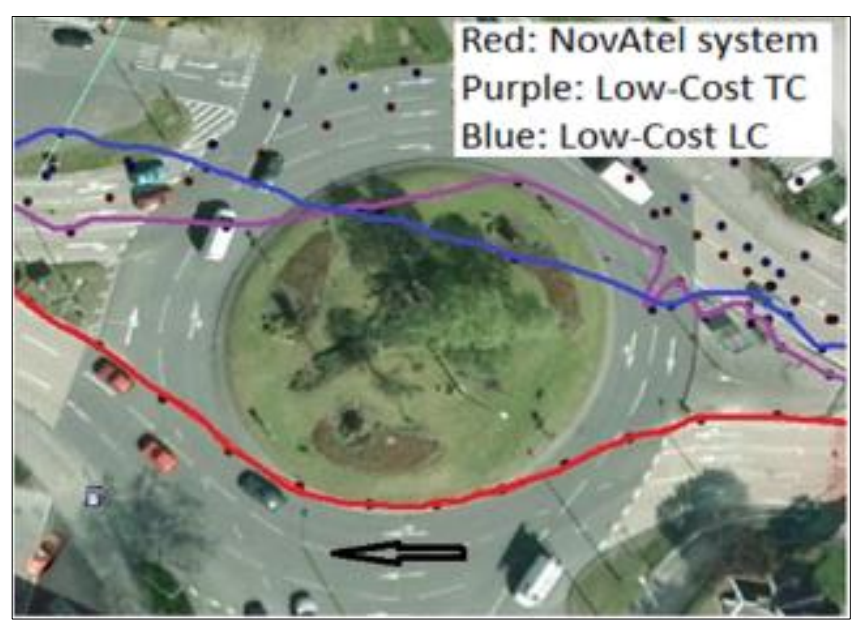

Figure 16 GPS off (Curvy path)

When comparing the performance of the low-cost GPS receiver individually with the low-cost navigation solutions, it has been noted that, in open sky as in figure (12), the individual carrier phase DGPS is very similar to the two navigation solutions of NovAtel and low-cost GPS/INS. This is because in loosely coupled integration, the navigation solution depends completely on GPS measurements to provide a 3D solution and the IMU measurements are just used to help the GPS for faster reacquisition. In the case of tightly coupled, the effects of IMU measurements in the solution is reduced due to the low weights given to the IMU measurements. As for the small differences between the individual GPS and the two navigation solutions, this can be attributed to the synchronizing between the time of individual GPS readings and the time of navigation solution obtained from GrafNav software. This is of course in addition to the differences in the performance of the single and dual frequency GPS receivers.

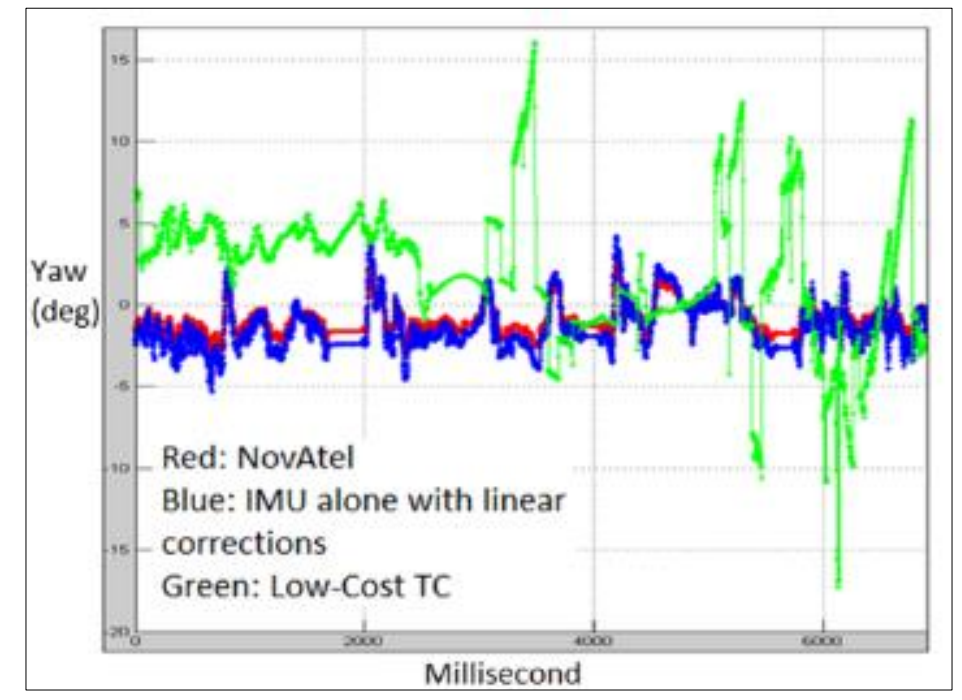

Figure 17 Yaw angle: Individual IMU and GPS/INS

In GPS/INS integration, GPS is used to correct the change in position provided by the INS. These relative positioning changes depend on the measurements of accelerometers and gyros. Therefore, when correcting the change in the position, values of both acceleration and rotation are corrected. The rotations provided by the gyros, as mentioned above, can be modeled and corrected to be more precise. The rotations achieved from the low-cost navigation solution and those of NovAtel solution have been compared to the rotations determined directly from the sensor and the results are illustrated in figure (17).

From the figure, it is clear that the rotations achieved individually from the sensor and those of NovAtel are close to each other and this is not the case with the low-cost navigation solution. This can be attributed to the effect of the ionosphere delay on the GPS measurements where in the case of single frequency GPS receivers, this effect is only mitigated using the base station corrections. This means that the precision of single frequency GPS measurements are less precise than those of dual frequency GPS positioning which use ionosphere-free method. The precision degree of 
GPS measurements plays a considerable role in the quality of the INS outputs where they are used as a main source for correcting the measurements of the accelerometers and gyros.

In the case of low-cost accelerometers such as those used in this test, even less precise GPS measurements are useful where the quality of the accelerometer measurements is much less precise than single frequency GPS and even A/C code positioning. However, the performance of the IMU in terms of rotations is good as shown in the previous section, especially when modeling and correcting the drifts. Therefore, when using less precise GPS measurements to bound the gyro drifts, no improvement can be seen. The other expected reason behind achieving less precise GPS measurements with the low-cost GPS receiver is the multipath effect where NovAtel GPS receiver has the ability to mitigate such effect using narrow correlation technique.

In conclusion, in this test, the integration of low-cost single frequency GPS with low-cost MEMS based INS has been evaluated in order to investigate whether this level of integration can help to overcome the limitations of the two systems and provide integrated system better than either on a stand-alone basis. Tests show that the main aim behind the integration of GPS/INS is difficult to be obtained in the case of integrating low-cost GPS/INS sensors. Tests show that such integration degrades the precision of the gyro measurements and may not add any improvements to the quality of the individual GPS positioning. It is recommended to investigate the possibility of enhancing the performance of this integration level using stand-alone double differencing career phase relative positioning or using real time visionbased navigation for simultaneous localization and mapping applications [8].

\section{Conclusion}

In this paper, the integration of low-cost L1-band based GPS receiver with low-cost MEMS based INS has been evaluated in order to investigate the advantages of such integration level over the individual performance of the two sensors. For reliable assessing, the low-cost single frequency GPS receiver and MEMS based INS used in this study have been tested individually to investigate the accuracy level can be obtained from such sensors; then, the integration of these two lowcost sensors has been evaluated comparing to the stand-alone performance of each sensor. The results show that lowcost single frequency GPS receivers are able to provide a comparable positioning accuracy level in both static and kinematic carrier phase DGPS solutions. The results show the capability of such sensors to provide accuracy of nearly 1 $\mathrm{cm}$ with static carrier phase DGPS and a mobile solution with 5 and $10 \mathrm{~cm}$ accuracy in plan and height, respectively. The tests show also the high possibility of these receivers to be used as a rover and a base station to carry out low-cost static and kinematic DGPS. In terms of A/C code positioning, 3D accuracy of a few meters can be obtained, deceasing significantly in multipath GPS environments.

As for the low-cost MEMS based INS (Microstrain 3DM-GX3-25 IMU), the sensor has been evaluated in terms of rotation quality, drift rate, and modeling and correcting gyro drifts. Tests show that the sensor can provide drift rate of about $0.29 \mathrm{deg} . / \mathrm{sec}$ and the angular drift of this sensor is quasi-linear. Instability in the performance of accelerometers has been recorded during the tests providing considerable errors even in stationary case. Simple linear filter has been designed and used to correct the angular drifts reducing the drift rate to round about $0.05 \mathrm{deg} . / \mathrm{sec}$.

The integration of low-cost single frequency GPS with low-cost MEMS based INS has been evaluated in order to investigate whether this level of integration can help to overcome the limitations of the two systems and provide integrated system better than either on a stand-alone basis. Tests show that the main aim behind the integration of GPS/INS may not be possible to be obtained in the case of integrating low-cost GPS/INS sensors. Tests show that such integration degrades the precision of the gyro measurements and may not add any improvements to the quality of the individual GPS positioning.

\section{Compliance with ethical standards}

\section{Acknowledgments}

I would like to thank everyone who contributed to the implementation of this work, as well as the staff of the Benghazi University and Nottingham University, who contributed with support and information to make this idea a success.

\section{References}

[1] Hofmann-Wellenhof B, Lichtenegger H, Wasle E. GNSS global navigation satellite systems: GPS, GLONASS, Galileo, and More. 2007. 
[2] Amami M. Low Cost Vision Based Personal Mobile Mapping System [Doctoral dissertation]. University of Nottingham, UK. 2015.

[3] Amami M. The Integration of Time-Based Single Frequency Double Differencing Carrier Phase GPS/ MicroElctromechanical System-Based INS. International Journal of Recent Advances and technology. Dec. 2018; 5(4): 43-56.

[4] Amami MM, Smith MJ, Kokkas N. Low Cost Vision Based Personal Mobile Mapping System. ISPRS- International Archives of The Photogrammetry, Remote Sensing and Spatial Information Sciences. 2014; XL-3/W1: 1-6. ISSN 2194-9034.

[5] Amami M. Enhancing Stand-Alone GPS Code Positioning Using Stand-Alone Double Differencing Carrier Phase Relative Positioning. Journal of Duhok University (Pure and Eng. Sciences). 2017; 20(1): 347-355.

[6] El-Rabbany A. Introduction to GPS: The global positioning system. Artech House. 2002.

[7] Titterton D, Weston J. Strapdown inertial navigation technology, 2nd ed. London: Institution of Engineering and Technology. 2004.

[8] Amami M. Speeding up SIFT, PCA-SIFT \& SURF Using Image Pyramid. Journal of Duhok University, [S.I]. July 2017; 20(1): 356-362. 